\title{
Adaptive Frequency domain Affine Projection Equalizer of MIMO SC-FDMA Based Overlap-Save Method
}

This paper was downloaded from TechRxiv (https://www.techrxiv.org).

LICENSE

CC BY 4.0

SUBMISSION DATE / POSTED DATE

$19-12-2021 / 21-12-2021$

CITATION

George, Maha (2021): Adaptive Frequency domain Affine Projection Equalizer of MIMO SC-FDMA Based Overlap-Save Method. TechRxiv. Preprint. https://doi.org/10.36227/techrxiv.17288000.v1

DOI

10.36227/techrxiv.17288000.v1 


\title{
Adaptive Frequency domain Affine Projection Equalizer of MIMO SC-FDMA Based Overlap-Save Method
}

\author{
Maha George Zia, Member, IEEE
}

\begin{abstract}
Minimum mean-square error frequency domain equalizer (MMSE-FDE) is a promising technique that can handle the effect of a multi-path propagation channel in multiple-input multiple-out (MIMO) systems and hence enhance the bit error rate (BER) performance in MIMO systems. MMSE-FDE is complex because its adapted weights require the calculation of channel state information (CSI) and signal-to interference plus noise power ratio (SINR). Both are considered as difficult tasks and important issues to obtain especially in long term evolution (LTE) and massive MIMO systems. To tackle these issues, an adaptive affine projection frequency domain equalizer (AAPFDE) used within overlap-save method (OLSM) is proposed where its adapted weights do not depend on CSI or SINR. The proposed AAPFDE within OLSM is used in conjunction with a turbo decoder to enhance the BER performance for $8 \times 8$ and 16x16 MIMO SCFDMA systems. Simulation results are compared and showed that MMSE-FDE outperforms the proposed AAPFDE in MIMO SCFDMA system. While the proposed AAPFDE is simple and achieves good BER performance, MMSE-FDE is more complex and still considered as optimum receiver.
\end{abstract}

Index Terms-Frequency domain equalizer, MIMO, overlapadd, overlap-save, SC-FDMA.

\section{INTRODUCTION}

$\mathrm{T}$ lo recover the transmitted signal in MIMO SC-FDMA system, time domain equalizers [1-3], and frequency domain equalizers are used [4-7]. Frequency domain equalizer (FDE) is preferred because it relays on Fast

Fourier Transform (FFT) that requires less computations which results in less round-off error or noise. The received signals in MIMO SC-FDMA system are coupled both in time and space and they need efficient receiver to enhance the BER performance. This can be realized by frequency domain equalization schemes [7-10].

The literature [8] adopts two frequency domain equalization

This paragraph of the first footnote will contain the date on which you submitted your paper for review, which is populated by IEEE. It is IEEE style to display support information, including sponsor and financial support acknowledgment, here and not in an acknowledgment section at the end of the article. For example, "This work was supported in part by the U.S. Department of Commerce under Grant BS123456." The name of the corresponding author appears after the financial information, e.g. (Corresponding author: M. Smith). Here you may also indicate if authors contributed equally or if there are co-first authors.

M. G. Zia is with Electrical Engineering Department, College of Engineering, Salahaddin University, Erbil, Iraq (email: maha.zia@su.edu.krd) schemes, the joint complex regularized zero forcing scheme (JCZF) and MMSE scheme. Both schemes were used to analyze and investigate the performance improvement of MIMO SCFDMA system under different carrier frequency offsets and channel models. Simulation results showed that JCZF scheme is highly competitive to MMSE scheme.

Different receiver algorithms and structures for MIMO SCFDMA were proposed in [9]. One of those structures is a frequency domain MMSE equalization with spherical decoding. Frame error rate performance was studied via simulations in different wireless channels and the receiver was designed with different stages to mitigate inter-symbol interference (ISI) and inter-antenna interference (IAI). The main disadvantage of this designed receiver is its complexity. MMSE linear frequency domain equalization was also investigated for SC-FDMA transmission over MIMO THz channel [10]. Simulation results showed that MMSE linear frequency domain equalization is promising for practical $\mathrm{THz}$ systems.

As was mentioned earlier, most of researchers depend on minimum mean-square error frequency domain equalizer (MMSE-FDE) as a promising technique that can handle the effect of a multi-path propagation channel in multiple-input multiple-out (MIMO) systems and have great BER performance improvement. The main disadvantage of MMSEFDE is its complexity because its adapted weights require the calculation of channel state information (CSI) and signal-to interference plus noise power ratio (SINR). Both are considered as difficult tasks and important issues to obtain especially in long term evolution (LTE) and massive MIMO systems.

In this paper, these issues are tackled by designing adaptive affine projection frequency domain equalizer (AAPFDE) used within overlap-save method (OLSM) where its adapted weights do not depend on CSI or SINR values. The

Second B. Author, Jr., was with Rice University, Houston, TX 77005 USA. $\mathrm{He}$ is now with the Department of Physics, Colorado State University, Fort Collins, CO 80523 USA (e-mail: author@lamar.colostate.edu).

Third C. Author is with the Electrical Engineering Department, University of Colorado, Boulder, CO 80309 USA, on leave from the National Research Institute for Metals, Tsukuba 305-0047, Japan (e-mail: author@nrim.go.jp).

Mentions of supplemental materials and animal/human rights statements can be included here.

Color versions of one or more of the figures in this article are available online at http://ieeexplore.ieee.org 
proposed AAPFDE within OLSM is used in conjunction with a turbo decoder to enhance the BER performance for $8 \times 8$ and 16x16 MIMO SC-FDMA systems in extended pedestrian A (EPA) channel. In summary, the motivation and contributions of this paper are:

- Design a moderate receiver without requiring difficult parameters to estimate or depend on like CSI and SINR, where these parameters depend mainly on the channel type and its characteristics.

- The moderate receiver designed in this paper depends on adaptive affine projection algorithm (APA) implemented in frequency domain equalizer within overlap-save method (OLSM). They are used in conjunction with a turbo decoding to enhance the BER performance of MIMO SC-FDMA system.

- $\quad$ The AAPFDE weights are derived and compared with MMSE-FDE weights.

- Demonstration of BER performances for $8 \times 8$ and 16x16 MIMO SC-FDMA systems in extended pedestrian A (EPA) channel using both MMSE-FDE and AAPFDE.

- Simulation results are compared and showed that MMSE-FDE outperforms the proposed AAPFDE in MIMO SC-FDMA system. While the proposed AAPFDE is simple and achieves good BER performance, MMSE-FDE is more complex and still considered as optimum receiver.

The remaining sections of this paper are organized as follows. The system structure is represented in section II. In section III, the proposed method based on affine projection algorithm is proposed to estimate the weights of the AAPFDE and to be compared with MMSE-FDE weights. The simulation results are introduced in section IV. Finally, the conclusion is presented in section $\mathrm{V}$.

\section{MIMO SC-FDMA SYSTEM STRUCTURE}

A proposed transceiver system model of MIMO SC-FDMA with $\mathrm{Nt} \times \mathrm{Nr}$ antennas is shown in Fig. 1. A frame of data bits of $\mathrm{i}$-th transmit antenna $(\mathrm{i}=1,2, . ., \mathrm{Nt})$ is encoded using a turbo encoder.

The coded bits are interleaved by using a random interleaver, and then they are QPSK modulated. A block of M data symbols is represented by $\boldsymbol{x}_{i}$, i.e., $\boldsymbol{x}_{i}=\left[x_{i, 0}, x_{i, 1}, \ldots, x_{i, M}\right]$, serial-toparallel converted and transformed to a frequency domain block $\boldsymbol{X}_{i}=\left[X_{i, 0}, X_{i, 1}, \ldots, X_{i, M}\right]$ using M-point fast Fourier transform (FFT).

After using localized mapping, $\boldsymbol{X}_{i}$ is mapped to $\mathrm{N}>\mathrm{M}$ subcarriers, i.e.

$$
\boldsymbol{S}_{i}=\boldsymbol{A} \boldsymbol{X}_{i}
$$

where $\boldsymbol{A}=N \times M$ is the source allocation matrix of the localized mapping. The block $\boldsymbol{S}_{i}$ is transformed to a time domain block $\boldsymbol{s}_{i}$ via N-point inverse fast Fourier transform (IFFT) and returns the transmitted SC-FDMA signal vector as:

$$
\boldsymbol{s}_{i}=\boldsymbol{F}_{N}^{H} \boldsymbol{A} \boldsymbol{F}_{M} \boldsymbol{x}_{i}=\boldsymbol{F}_{N}^{H} \boldsymbol{A} X_{i}
$$

where $(.)^{\mathrm{H}}$ denotes the Hermitian operator. $\boldsymbol{F}_{N}$ and $\boldsymbol{F}_{M}$ are $N \times N$ and $M \times M$ FFT matrices, respectively.

Instead of using a cyclic prefix (cp) in the proposed model, circular sub-channel matrices $\boldsymbol{H}_{i, j}$ are assumed to achieve circular property in the transmission. Also, the sum of transmit power is assumed to be constant regardless of the number of transmit antennas.

The received signal vector of $\mathrm{j}$-th receiver antenna $(\mathrm{j}=1$, $2, \ldots, \mathrm{Nr}$ ) is given by:

$$
\left[\begin{array}{c}
\boldsymbol{y}_{1} \\
\vdots \\
\boldsymbol{y}_{N r}
\end{array}\right]=\left[\begin{array}{ccc}
\mathcal{H}_{1,1} & \ldots & \mathcal{H}_{1, N t} \\
\vdots & \ddots & \vdots \\
\mathcal{H}_{N r, 1} & \ldots & \mathcal{H}_{N r, N t}
\end{array}\right]\left[\begin{array}{c}
\boldsymbol{s}_{1} \\
\vdots \\
\boldsymbol{s}_{N t}
\end{array}\right]+\left[\begin{array}{c}
\boldsymbol{\eta}_{1} \\
\vdots \\
\boldsymbol{\eta}_{N r}
\end{array}\right]
$$

where $\boldsymbol{H}_{i, j}$ is the frequency selective fading channel matrix between the $\mathrm{i}$-th transmit antenna and $\mathrm{j}$-th received antenna. The $\boldsymbol{\eta}_{N r}$ is a complex valued additive white Gaussian noise with zero mean and unit variance. Assuming there are $\mathrm{L} \leq \mathrm{N}$ taps of the frequency selective channel, and due to circular property, each sub-matrix in (3) can be written as:

$$
\mathcal{H}_{i, j}=\left[\begin{array}{ccccc}
h_{i, j}^{1} & h_{i, j}^{N} & \ldots & h_{i, j}^{3} & h_{i, j}^{2} \\
h_{i, j}^{2} & h_{i, j}^{1} & \ldots & h_{i, j}^{4} & h_{i, j}^{3} \\
\vdots & \ddots & \ddots & \ddots & \vdots \\
h_{i, j}^{N} & h_{i, j}^{N-1} & \ldots & h_{i, j}^{2} & h_{i, j}^{1}
\end{array}\right]
$$

In (4), all entries $h_{i, j}^{n}$ with $\mathrm{n}>\mathrm{L}$ are zero. In this paper, an extended pedestrian A (EPA) channel is assumed with $L$-paths frequency selective fading channel and its impulse response can be expressed as [29]:

$$
h(t-\tau)=\sum_{l=1}^{L} \alpha_{l} \delta\left(t-\tau_{l}\right)
$$

where $\alpha_{l}$ is the complex value path gain of the $(l=1,2, \ldots L)$ path between $\mathrm{i}$-th transmit antenna and $\mathrm{j}$-th received antenna. $\tau_{l}$ is the $l$-th path delay time.

At the receiver, after $\mathrm{N}$-point $\mathrm{FFT}$ and demapping, the received signal vector in frequency domain is represents by:

$$
\left[\begin{array}{c}
\boldsymbol{Y}_{1} \\
\vdots \\
\boldsymbol{Y}_{N r}
\end{array}\right]=\left[\begin{array}{ccc}
\boldsymbol{H}_{1,1} & \ldots & \boldsymbol{H}_{1, N t} \\
\vdots & \ddots & \vdots \\
\boldsymbol{H}_{N r, 1} & \ldots & \boldsymbol{H}_{N r, N t}
\end{array}\right]\left[\begin{array}{c}
\boldsymbol{S}_{1} \\
\vdots \\
\boldsymbol{S}_{N t}
\end{array}\right]+\left[\begin{array}{c}
\boldsymbol{\Pi}_{1} \\
\vdots \\
\boldsymbol{\Pi}_{N r}
\end{array}\right]
$$

where $\boldsymbol{H}_{i, j}$ is $M \times M$ channel gain diagonal matrix, $\boldsymbol{S}_{i}$, and $\boldsymbol{\Pi}_{j}$ are the signal component, and noise component, respectively.

\section{A. Turbo decoding in conjunction with the overlap-save method (OLSM) block}

The aim is to design the overlap-save method (OLSM) block diagram in which the adaptive affine projection FDE is 
$>$ REPLACE THIS LINE WITH YOUR MANUSCRIPT ID NUMBER (DOUBLE-CLICK HERE TO EDIT) <

considered as the main part of it. The OLSM block diagram is shown in Fig. 2. The received SC-FDMA signal vector $\boldsymbol{Y}_{j}$ is transformed again to time domain symbols using M-point IFFT and enters overlap-save method (OLSM) block [11-13]. The time domain vector at point (a) is divided into a sequence of $B$ symbol blocks $\left(B \leq \mathrm{N}_{B}\right)$ with certain overlap of length $N_{B} \cdot N_{B}$ is the FFT size used to convert these blocks to a frequency domain. The frequency domain signal block is represented by:

$$
\boldsymbol{R}_{\boldsymbol{j}}(k)=\mathbf{H}_{i, j}(k) \mathbf{S}(k)+\boldsymbol{\Delta}_{\boldsymbol{j}}(k), \quad k=0,1, \ldots, N_{B}(7)
$$

where $\mathbf{H}_{i, j}(k), \mathbf{S}(k)$, and $\boldsymbol{\Delta}_{\boldsymbol{j}}(k)$ are respectively the channel gain, the signal component, and noise component due to AWGN. Now, each frequency component $\boldsymbol{R}_{j}(k)$ is multiplied by the adaptive affine projection frequency domain equalizer (AAPFDE) weight $\mathbf{w}_{j}(k)$ :

$$
\boldsymbol{R}_{j}(k)=\sum_{j} \mathbf{w}_{j}(k) \boldsymbol{R}_{j}(k) .
$$

Next, the equalized symbol blocks $\boldsymbol{\mathcal { R }}_{j}(k)$ are transformed into time domain by using $N_{B}$-IFFT where the processed data blocks are combined to form $\boldsymbol{D}_{j}$ vector at point (b). The vector $\boldsymbol{D}_{j}$ is now entered to QPSK demodulator. The output of the demodulator is de-interleaved and is input to APP turbo decoder [14-17].

The log likelihood ratios (LLR) of information bit $b_{k}$, and coded bit $c_{k}$ for $k=0,1, \ldots, v-1$, of APP turbo decoder are given by:

$$
\begin{gathered}
\Lambda\left(b_{k}\right)=\ln \frac{\sum_{b_{k} \in s_{1}}\left(p\left(b_{k}=1\right) p\left(\widehat{b} \mid b_{k}=1\right)\right)}{\sum_{b_{k} \in s_{0}}\left(p\left(b_{k}=0\right) p\left(\widehat{b} \mid b_{k}=0\right)\right)} . \\
\Lambda\left(c_{k}\right)=\ln \frac{\sum_{c_{k} \in s_{1}}\left(p\left(c_{k}=1\right) p\left(\hat{c} \mid c_{h}=1\right)\right)}{\sum_{c_{k} \in s_{0}}\left(p\left(c_{k}=0\right) p\left(\hat{c} \mid c_{h}=0\right)\right)} .
\end{gathered}
$$

where, $p\left(b_{k}=0\right), p\left(b_{k}=1\right), p\left(c_{k}=0\right)$, and $p\left(c_{k}=1\right)$ denote the respective a-priori probabilities for information bit $b_{k}$ and coded bit $c_{k}$.

The conditional probability densities of the received signal sample $\widehat{b}$ and coded signal sample $\hat{c}$ given the transmission of " 0 " at the current position $k$ are denoted by $p\left(\widehat{b} \mid b_{k}=0\right)$, and $p\left(\hat{c} \mid c_{k}=0\right)$ respectively.

The conditional probability densities of the received signal sample $\widehat{b}$ and coded signal sample $\hat{c}$ given the transmission of "1" at the current position $k$ are denoted by $p\left(\widehat{b} \mid b_{k}=1\right)$ and $p\left(\hat{c} \mid c_{k}=1\right)$ respectively. $s_{0}$ and $s_{1}$ denote the respective set of modulation symbols comprising a " 0 " / "1" at current position $k$.

The a priori log-likelihood ratio (LLR) of the output coded bits is fed back, interleaved, and converted to a frequency domain via FFT as $\boldsymbol{C}_{\boldsymbol{j}}$ vector which is subtracted from the frequency domain vector $\boldsymbol{V}_{j}$ to form as an error vector (e) to be applied to AAPFDE.

\section{PROPOSED METHOD}

The proposed method is based on designing a receiver based on overlap-save method (OLSM) of MIMO SC-FDMA system as shown in Fig. 1 and Fig. 2. The main part of OLSM is the adaptive affine projection frequency domain equalizer (AAPFDE) [18-22]. This section focuses on finding the weights of AAPFDE and compare them the with the weights of MMSEFDE.

Let the input vectors be $\mathbf{x}(n), \mathbf{x}(n-1), \ldots, \mathbf{x}(n-P o+1)$ and the wanted output samples to be $\mathrm{d}(n), \mathrm{d}(n-1), \ldots, \mathrm{d}(n-$ $P o+1)$, where $P o$ is the projection order. To minimize the squared Euclidian norm of the difference between the updated weight vector $\mathbf{w}(n+1)$, and the previous weight vector $\mathbf{w}(n)$ is given by:

$$
\boldsymbol{\varsigma}(n)=\mathbf{w}(n+1)-\mathbf{w}(n) .
$$

Equation (11) has the constrains,

$$
\mathbf{w}^{T}(n+1) \mathbf{x}(n-\mathrm{k})=d(n-\mathrm{k}), \mathrm{k}=1,2, \ldots, P o
$$

Where (.) $)^{\mathrm{T}}$ denotes transpose operation. Lagrange multipliers $\mathfrak{L}^{c}$ are used to solve (12). Defining $\mathbf{X}(n)$ as $N_{F} \times P o$ matrix, the filter length is $N_{F}$ and $\mathbf{d}(n)$ is a column vector of length $P o$; then (12) becomes:

$$
\mathbf{X}^{T}(n) \mathbf{w}(n+1)=\mathbf{d}(n)
$$

Defining:

$$
\mathfrak{L}^{c}=\|\mathbf{w}-\mathbf{w}(n)\|^{2}+\left(\mathbf{X}^{T}(\mathrm{n}) \mathbf{w}-\mathbf{d}(n)\right)^{T} \boldsymbol{\lambda} .
$$

Where $\lambda$ is a column vector of Lagrange multipliers $\lambda_{1}, \lambda_{2}, \ldots, \lambda_{P O}$. Equation (12) can be solved using:

$$
\begin{aligned}
& \nabla_{\mathbf{w}} \mathfrak{L}^{c}=0 . \\
& \nabla_{\Lambda} \mathfrak{L}^{c}=0 .
\end{aligned}
$$

Noting that $\|\mathbf{w}-\mathbf{w}(n)\|^{2}=(\mathbf{w}-\mathbf{w}(n))^{T}(\mathbf{w}-\mathbf{w}(n))$ and substituting (14) in (15) and (16), yield:

$$
\begin{gathered}
2(\mathbf{w}(n+1)-\mathbf{w}(n))+\mathbf{X}(n) \lambda=0 . \\
\mathbf{X}^{T}(n) \mathbf{w}(n+1)-\mathbf{d}(n)=0 .
\end{gathered}
$$

It can be noticed that the solution of (17) and (18) yield $\mathbf{w}(n)=\mathbf{w}(\mathrm{n}+1)$. Multiplying (17) by $\mathbf{X}^{T}(n)$, using (18) and defining:

$$
\mathbf{e}(n)=\mathbf{d}(n)-\mathbf{X}^{T}(n) \mathbf{w}(n) .
$$

Then:

$$
\lambda=-2\left(\mathbf{X}^{\mathbf{T}}(n) \mathbf{X}(n)\right)^{-1} \mathbf{e}(n) .
$$


$>$ REPLACE THIS LINE WITH YOUR MANUSCRIPT ID NUMBER (DOUBLE-CLICK HERE TO EDIT) <

Substituting (20) in (17) and rearranging the result yield:

$$
\mathbf{w}(n+1)=\mathbf{w}(n)+\mathbf{X}(n)\left(\mathbf{X}^{\mathbf{T}}(n) \mathbf{X}(n)\right)^{-1} \mathbf{e}(n) \cdot(21)
$$

In practice, (21) can be written as:

$$
\mathbf{w}(n+1)=\mathbf{w}(n)+\mu \mathbf{X}(n)\left(\mathbf{X}^{\mathbf{T}}(n) \mathbf{X}(n)+\psi \mathbf{I}\right)^{-1} \mathbf{e}(n) .
$$

where $\mu$ is the step size parameter, $\mathbf{I}$ is $P o \times P o$ identity matrix, and $\psi$ is a small positive constant to ensure the numerical stability of the algorithm when $\mathbf{X}^{\mathbf{T}}(n) \mathbf{X}(n)$ is near singular matrix.

According to pervious prove, if $P o=1$, the APA is just normalized least mean square (NLMS) algorithm. As $P o$ increases, APA offers a good convergence improvement compared to NLMS algorithm but with large number of computations that becomes as a heavy burden for real time processing [22]. It is still less complex compared to MMSE algorithm where the tap weight vector adaptation $\boldsymbol{W}(\boldsymbol{K})$ in MMSE-FDE depends on channel state information and signalto interference plus noise power ratio (SINR) as described below [23]:

$$
\boldsymbol{W}(\boldsymbol{K})=\frac{\boldsymbol{H}^{\star}(\boldsymbol{K})}{|\boldsymbol{H}(\boldsymbol{K})|^{2}+\mathcal{\varkappa}^{-1}}
$$

Where, $H(K)$ is channel state information (CSI), and $\mathcal{\varkappa}^{-1}$ is signal-to-interference plus noise ratio (SINR).

\section{RESULTS AND DISCUSSION}

The bit error rate (BER) performances of MIMO SC-FDMA system using the proposed AAFDE and MMSE-FDE are evaluated by simulations. The turbo encoder is of rate $1 / 2$ and its generator polynomial is represented as (5, [23 35], 23). QPSK data modulation is used. The extended pedestrian A (EPA) model is used as a channel model [29]. It has seven Rayleigh fading taps at delays of $0,30,70,90,110,190$, and $410 \mathrm{~ns}$, with relative power of $0,-1,-2,-3,-8,-17.2$, and -20.8 $\mathrm{dB}$, respectively. Five iterations are performed for both AAPFDE and turbo decoder. Table I summarizes simulation parameters.

Block LU decomposition [ 24-28] was used to reduce the execution time of the matrix inversion of the adapted weights of both equalizers. The execution time of the proposed system using AAPFDE and MMSE-FDE was measured without and with block LU decomposition as listed in Table II. By comparing (22) and (23), it can be noticed that the execution time without and with block LU decomposition for AAPFDE is less than MMSE-FDE because MMSE-FDE requires more time to find CSI as well as SINR.

Figure 3 shows the BER performances using three different step size values with projection order $P o=2$ of AAPFDE. The FFT size in OLSM for both AAPFDE and MMSE-FDE is $\mathrm{N}_{B}=$ 32. Coding gain of $2 \mathrm{~dB}$ is observed at $\mathrm{BER}=5 \times 10^{-2}$ between $8 \times 8$ and 16x16 MIMO SC-FDMA using MMSE-FDE. Also, at $\mathrm{BER}=5 \times 10^{-2}$ for AAPFDE, coding gain values of $1.5 \mathrm{~dB}, 2$
$\mathrm{dB}$ and $1.75 \mathrm{~dB}$ are observed for $\mu=0.01,0.1$ and 0.5 respectively between $8 \times 8$ and 16x16 MIMO SC-FDMA systems. It is seen that better performances can be obtained when using AAPFDE with small values of $\mu$. Although good BER performances are obtained when $\mu=0.01$ for AAPFDE, the BER performances of MMSE-FDE is much better than the BER performances of AAPFDE for 8x8 and 16x16 MIMO SCFDMA systems.

Three different projection order values with step size $\mu=$ 0.1 of AAPFDE are illustrated in Figure.4. The FFT size in OLSM for both AAPFDE and MMSE-FDE is $\mathrm{N}_{B}=32$. Coding gain of $2 \mathrm{~dB}$ is observed at $\mathrm{BER}=5 \times 10^{-2}$ using MMSE-FDE. The worse performance of AAPFDE is obtained at $\mathrm{BER}=5 \times 10^{-2}$ and $P o=1$, here the AAPFDE works as adaptive normalized least mean square (NLMS) equalizer with a performance gain of $1.25 \mathrm{~dB}$ obtained between $8 \times 8$ and $16 \times 16$ MIMO SC-FDMA systems. At the same $\mathrm{BER}=5 \times 10^{-2}$, better performances of AAPFDE can be obtained for higher order of $P o$ values where, coding gains of $1 \mathrm{~dB}$ and $2 \mathrm{~dB}$ are obtained at $P o=3$ and $P o=2$ respectively. By comparison of simulation results, MMSE-FDE still behaves better than AAPFDE (with $P o=3$ ) for $8 \times 8$ and 16x16 MIMO SC-FDMA systems.

Two $\mathrm{N}_{\mathrm{B}}$ values of 32 and 64 are chosen for both MMSE-FDE and AAPFDE as shown in Figure 5. These values are applied for $8 \times 8$ and 16x16 MIMO SC-FDMA systems. The AAPFDE is applied with step size value $\mu=0.1$ and projection order $P o=2$. At BER $=5 \times 10^{-2}$, coding gains of values $2 \mathrm{~dB}$, and $2 \mathrm{~dB}$ are obtained with $\mathrm{N}_{\mathrm{B}}=32$ for MMSE-FDE and AAPFDE, respectively. The system performs worse at $N_{B}=64$ with coding gains of values $1 \mathrm{~dB}$, and $2 \mathrm{~dB}$ for MMSE-FDE and AAPFDE, respectively. It can be noticed that when $\mathrm{N}_{B}$ is small, better performances can be obtained. MMSE-FDE behaves much better than the proposed AAPFDE for both $\mathrm{N}_{\mathrm{B}}$ values.

\section{CONCLUSION}

Throughout this work, a moderate receiver was designed that depends on adaptive affine projection algorithm (APA) implemented in frequency domain equalizer as AAPFDE within overlap-save method (OLSM). Also, a turbo decoder was used in the proposed receiver to enhance the BER performance in $8 \times 8$ and 16x16 MIMO SC-FDMA systems. It was presented that the proposed AAPFDE weights are much simpler compared with MMSE-FDE weights because they do not depend on channel state information (CSI) or signal -tointerference noise power ratio (SINR). These two issues are difficult to obtain especially in LTE and massive MIMO systems.

Demonstration of BER performances for both equalizers in 8x8 and 16x16 MIMO SC-FDMA systems were evaluated by simulations. Block LU decomposition was used that reduces the execution time of the matrix inversion of both equalizers.

Different parameters of AAPFDE affect the BER performances were demonstrated; they are step size $\mu$, and projection order $\mathrm{Po}$. It was shown by simulation that $8 \times 8$ MIMO SC-FDMA has better performance than 16x16 MIMO SC-FDMA system using AAPFDE for small value of $\mu$, and 
moderate value of $P o$.

For both equalizers, two $\mathrm{N}_{B}$ values of 32 and 64 were considered. It was shown by simulation that even for $\mathrm{N}_{B}=64$ , MMSE-FDE provides good performance than AAPFDE with $\mathrm{N}_{B}=32$.

Finally, according to the mentioned above, MMSE-FDE outperforms the proposed AAPFDE in MIMO SC-FDMA system. While the proposed AAPFDE is simple and achieves good BER performance, MMSE-FDE is more complex and still considered as optimum receiver.

\section{REFERENCES}

[1] B. Dhivagar, K. Kuchi, and K. Giridhar, "An Iterative DFE Receiver for MIMO SC-FDMA Uplink," IEEE Communications Letters, vol.18, no. 12, pp. 2141-2144, Dec. 2014.

[2] K. Selvaraj, et al., "Low Complexity Linear Detection for Uplink Multiuser MIMO SC-FDMA Systems," Wireless Personal Communications, Springer, vol. 112, pp. 631-649, Jan. 2020.

[3] R. Zedka, T. Götthans, and R. Maršálek., "Spectral Efficient Time-Domain Equalization Single-Carrier System," in Proceeding IEEE $31^{\text {st }}$ International Conference Radioelectronika, Brno, Czech Republic, pp.1-5, April 2021.

[4] N. Iqbal, et al., "Adaptive Frequency-Domain RLS DFE for Uplink MIMO SC-FDMA," IEEE Transaction on Vehicular Technology, vol. 64, no. 7, pp. 2819-2833, July 2015.

[5] H-S. Wang, Fang-Biau Heng and Yu-Kuan Chang," Novel Turbo Receiver for MU-MIMO SC-FDMA System," ETRI Journal, vol. 40, no. 3, pp. 309317, April 2018.

[6] M. Anbar, et al., "Iterative SC-FDMA Frequency Domain Equalization and phase Noise Mitigation," IEEE in Proceeding IEEE International Symposium on Intelligent Signal Processing and Communications Systems (ISPACS), Ishigaki, Japan, pp. 91-95, Nov. 2018.

[7] S. Sardar, et al.," A Framework for Iterative frequency Domain EP-Based Receiver Design," IEEE Transactions on Communications, vol. 66, no. 12, pp. 6478-6493, Dec. 2018.

[8] M. Mustafa, et al., "MIMO SC-FDMA System Performance Investigation under Realistic channel Models," in Proceeding IEEE $36^{\text {th }}$ National Radio Science Conference (NRSC), Port Said, Egypt, pp. 145-152, April 2019.

[9] T. Hänninen, J. Ketonen and M. Juntti," MIMO Detector for LTE/LTE-A Uplink Receiver," Springer, Journal of Signal Processing Systems, vol. 91, no. 5, pp.423-435, May 2019.

[10] V. Schram, et al., "Concepts for Indoor THz Communications Systems," in Proceeding IEEE $3^{\text {rd }}$ International Workshop on Mobile Terahertz Systems (IWMTS), Esseni, Germany, pp. 1-5, July 2020.

[11] M. Najam, et al., "Hardware Implementation of Overlap-Save Based Fading Channel Emulator," IEEE Transactions on Circuits and Systems II, vol. 68, no. 3, pp.918-922, Sept. 2020.

[12] C. Li, H. K. Kwan, and X. Qin, "Revisiting Linear Convolution, Circular Convolution and Their Related Methods," in Proc. IEEE $13^{\text {th }}$ International Congress on Image and Signal Processing, BioMedical Engineering and Informatics, Chengdu, China, pp. 1124-1131, Oct. 2020.

[13] C. Bae and O. Gustafsson," Overlap-Save Commutators for High-Speed Streaming Data Filtering," in Proceeding IEEE International Symposium on Circuits and Systems (ISCAS), Daegu, South Korea, pp. 1-5, April 2021.

[14] M. Tomlinson, et al.," Error-Correction Codeing and Decoding: Bounds, Codes, Decoders, Analysis and Application," Springer, 2017.

[15] F. Shahin, et al., "Performance Analysis of High Throughput MAP Decoder for Turbo Codes and Self Concatenated Convolutional Codes, "IEEE Access, vol.7, pp. 138979-138093, Oct. 2019.

[16] N. Minallah, et al., "Analysis of Near-Capacity Iterative Decoding Schemes for Wireless Communications Using EXIT Charts," IEEE Access, vol.8, pp. 124424-124436, July 2020.

[17] M. Qiu, et al., "Analysis and Design of Partially- and Partially ParityCoupled Turbo Codes," IEEE Transactions on Communications, vol. 69, no. 4, pp. 2107-2122, April 2021.
[18] K. Ozeki, "Theory of Affine Projection Algorithms for Adaptive Filtering," Springer, Japan, 2016.

[19] Z. Zheng, Z. Liu, and Y. Dong, "Steady-State and Tracking Analysis of the Improved Proportionate Affine Projection Algorithm," IEEE Transactions on Circuits and Systems II, vol. 65, no. 11, pp. 1793-1797, Nov. 2018.

[20] L. Shi, et al., "Variable Step-Size Widely Linear Complex-Valued Affine Projection Algorithm and Performance Analysis," IEEE Transactions on Signal Processing, vol. 68, no. pp. 5940-5953, Oct. 2020.

[21] S. Sitjongsataporn, S. Prongunch, and T. Wiangtong,"Diffusion Affine Projection Sign Algorithm based on QR-Decomposition," in Proceeding IEEE $9^{\text {th }}$ International Electrical Engineering Congress (iEECON), Pattaya, Thailand, pp. 373-376, May 2021.

[22] M. Vilà; C. Alejandro López, and J. Riba,"Affine Projection Subspace Tracking," in Proceeding IEEE International Conference on Acoustics, Speech and Signal Processing, Toronto, Canada, pp. 3705-3709, June 2021.

[23] A Frequency Domain Joint MMSE-SIC Equalizer for MIMO SC-FDMA LTE-A Uplink," in Proceeding IEEE International Conference on Electronics and Communication Systems (ICECS), Coimbatore, India, pp. 253-256, Feb. 2014.

[24] J. G. Gentle," Matrix Algebra: Theory, Computations and Applications in Statistics," Springer, 2017.

[25] J. Liu, et al.," Spark-Based Large-Scale Matrix Inversion for Big Data Processing," IEEE Access, vol. 4, pp. 10042-10050, Jan. 2017.

[26] A. A. Muller, et al., "Computational Cost Reduction for $\mathrm{N}+2$ Order Coupling Matrix Synthesis Based on Desnanot-Jaccobi Identity," IEEE Access, vol. 4, pp.2166-2176, May 2016.

[27] L. Ma, et al.," QR Decomposition-Based Matrix Inversion for High Performance Embeded MIMO Receivers," IEEE Transaction on Signal Processing, vol. 59, no. 4, pp. 1858-1867, April 2011.

[28] M. A. M. Albreem, et al., "On Approximate Matrix Inversion Methods for Massive MIMO Detectors," IEEE Wireless Communications and Networking Conference (WCNC), Marrakesh, Morocco, pp.1-6, 2019

[29] ETSI TS 136116 V14.3.0 (2017)," LTE; Evolved Universal Terrestrial Radio Access (E-UTRA), Relay Radio Transmission and Reception". 


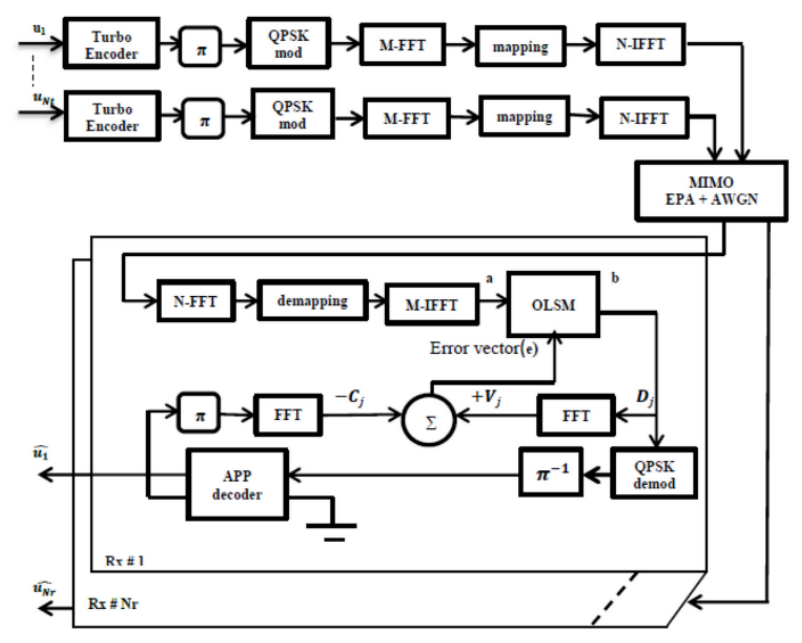

Fig.1 Proposed model of MIMO SC-FDMA

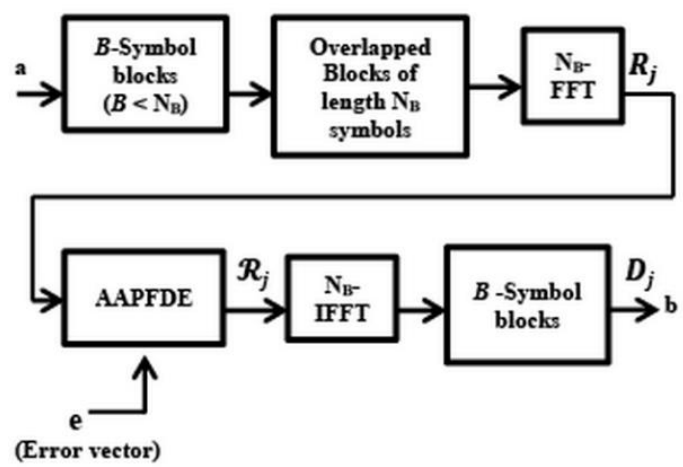

Fig.2 Over-lap save method block diagram

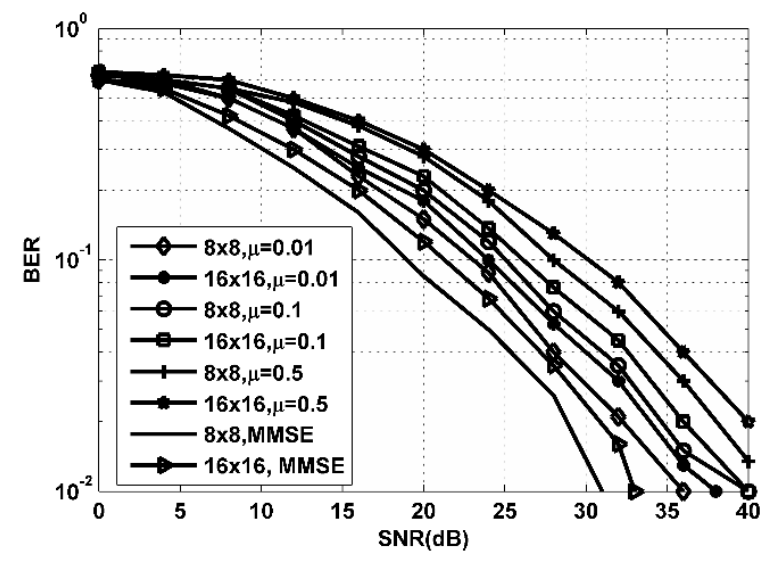

Figure 3. BER performances of $8 \times 8$ and $16 \times 16$ MIMO SCFDMA systems using MMSE-FDE and AAPFDE (applied with different step size values, and $\mathrm{Po}=2$ ). Both equalizers have $\mathrm{N}_{\mathrm{B}}$ $=32$.

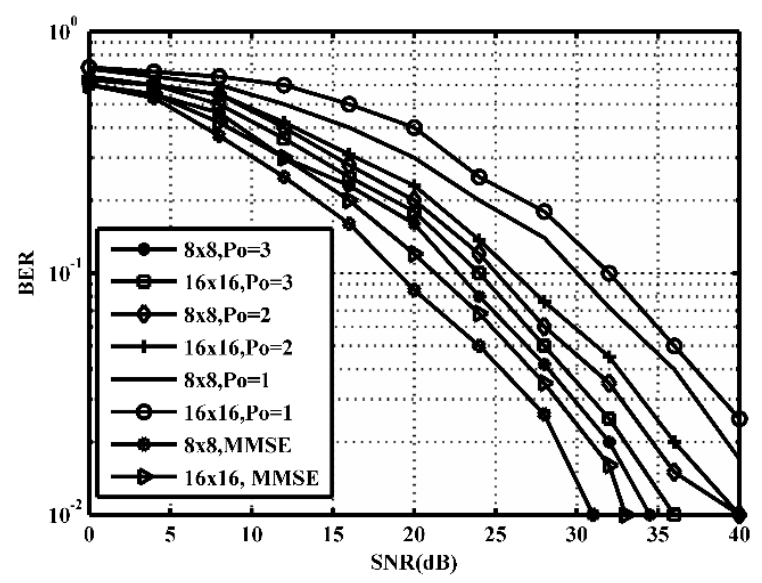

Figure 4. BER performances of $8 \times 8$ and $16 \times 16$ MIMO SCFDMA systems using MMSE-FDE and AAPFDE (applied with different projection orders and $\mu=0.1$ ). Both equalizers have $\mathrm{N}_{\mathrm{B}}=32$.

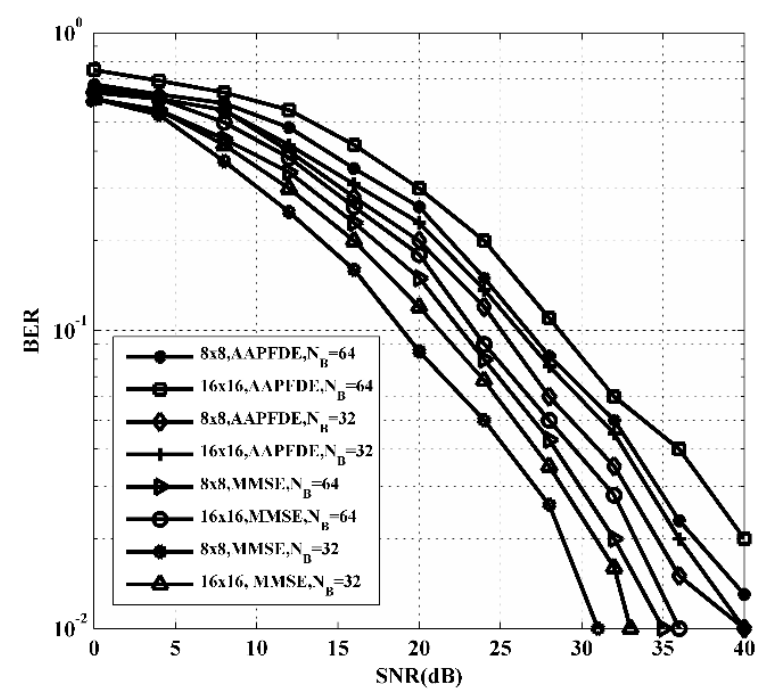

Figure 5. BER performances of $8 \times 8$ and $16 \times 16$ MIMO SCFDMA system using different $\mathrm{N}_{B}$ values for MMSE-FDE and AAPFDE (with $\mu=0.1$ and $\mathrm{Po}=2$ ). 
$>$ REPLACE THIS LINE WITH YOUR MANUSCRIPT ID NUMBER (DOUBLE-CLICK HERE TO EDIT) <

Table I Simulation Parameters

\begin{tabular}{|l|l|}
\hline Parameter & Value \\
\hline System bandwidth & $5 \mathrm{MHz}$ \\
\hline Interleaver type & random \\
\hline SC-FDMA mapping & Localized \\
\hline Channel coding & Turbo coding, rate $1 / 2$ \\
\hline Channel model & EPA \\
\hline MIMO Technique & Transmit diversity \\
\hline Number of transmitter antennas & 2,4 \\
\hline Number of receiver antennas & 2,4 \\
\hline FFT size for overlap-save method $\mathbf{N}_{\boldsymbol{B}}$ & 32,64 \\
\hline Adaptive FDE algorithm & Affine Projection, MMSE \\
\hline Step size of AAPFDE & $0.1,0.01,0.5$ \\
\hline Projection order $(\boldsymbol{M})$ of AAPFDE & $1,2,3$ \\
\hline
\end{tabular}

Table II. Execution time without and with Block LU

Decomposition

\begin{tabular}{|l|c|c|}
\hline \multicolumn{1}{|c|}{$\begin{array}{c}\text { System Type with } \\
\mathbf{N}_{\boldsymbol{B}}=\mathbf{3 2}\end{array}$} & $\begin{array}{c}\text { without Block } \\
\text { LU } \begin{array}{c}\text { Decomposition } \\
\text { (sec.) }\end{array}\end{array}$ & $\begin{array}{c}\text { with Block LU } \\
\text { Decomposition } \\
\text { (sec.) }\end{array}$ \\
\hline $\begin{array}{l}\text { 8x8 MIMO SC-FDMA } \\
\text { using AAPFD }\end{array}$ & 301.84 & 161.49 \\
\hline $\begin{array}{l}\text { 16x16 MIMO SC-FDMA } \\
\text { using AAPFDE }\end{array}$ & 389.09 & 196.25 \\
\hline $\begin{array}{l}\text { 8x8 MIMO SC-FDMA } \\
\text { using MMSE-FDE }\end{array}$ & 408.34 & 218.06 \\
\hline $\begin{array}{l}\text { 16x16 MIMO SC-FDMA } \\
\text { using MMSE-FDE }\end{array}$ & 557.12 & 323.65 \\
\hline
\end{tabular}

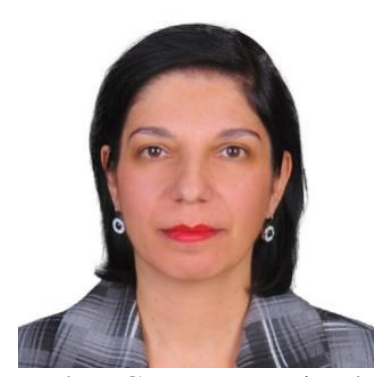

Maha George received the B.S. degree in Electrical Engineering from Baghdad University in Baghdad, Iraq, in 1986, and received the M.S. degree in Electronics and Communication Engineering from Baghdad University in 1992. Currently she is working as Assistant Professor in Electrical Engineering Department - College of Engineering-Salahaddin University -Erbil, Iraq. Her research interests include data communications, multicarrier systems, channel coding, MIMO systems, LTE, mobile communications, and digital signal processing. She is also IEEE member since 2007. 